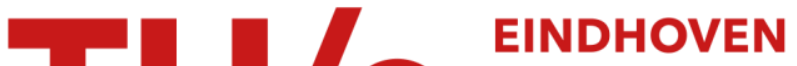

\section{A molecular model for cohesive slip at polymer melt/solid interfaces}

Citation for published version (APA):

Tchesnokov, M. A., Molenaar, J., Slot, J. J. M., \& Stepanyan, R. (2005). A molecular model for cohesive slip at polymer melt/solid interfaces. Journal of Chemical Physics, 122(21), 214711-1/12.

https://doi.org/10.1063/1.1915327

DOI:

10.1063/1.1915327

Document status and date:

Published: 01/01/2005

Document Version:

Publisher's PDF, also known as Version of Record (includes final page, issue and volume numbers)

Please check the document version of this publication:

- A submitted manuscript is the version of the article upon submission and before peer-review. There can be important differences between the submitted version and the official published version of record. People interested in the research are advised to contact the author for the final version of the publication, or visit the $\mathrm{DOI}$ to the publisher's website.

- The final author version and the galley proof are versions of the publication after peer review.

- The final published version features the final layout of the paper including the volume, issue and page numbers.

Link to publication

\section{General rights}

Copyright and moral rights for the publications made accessible in the public portal are retained by the authors and/or other copyright owners and it is a condition of accessing publications that users recognise and abide by the legal requirements associated with these rights.

- Users may download and print one copy of any publication from the public portal for the purpose of private study or research.

- You may not further distribute the material or use it for any profit-making activity or commercial gain

- You may freely distribute the URL identifying the publication in the public portal.

If the publication is distributed under the terms of Article $25 \mathrm{fa}$ of the Dutch Copyright Act, indicated by the "Taverne" license above, please follow below link for the End User Agreement:

www.tue.nl/taverne

Take down policy

If you believe that this document breaches copyright please contact us at:

openaccess@tue.nl

providing details and we will investigate your claim. 


\title{
A molecular model for cohesive slip at polymer melt/solid interfaces
}

\author{
M. A. Tchesnokov a) and J. Molenaar ${ }^{\text {b) }}$ \\ Department of Applied Mathematics, University of Twente, P.O. Box 217, 7500 AE Enschede, Netherlands \\ J. J. M. Slot ${ }^{\mathrm{C})}$ and R. Stepanyan \\ Department of Applied Physics, University of Twente, P.O. Box 217, 7500 AE Enschede, Netherlands
}

(Received 7 September 2004; accepted 24 March 2005; published online 6 June 2005)

\begin{abstract}
A molecular model is proposed which predicts wall slip by disentanglement of polymer chains adsorbed on a wall from those in the polymer bulk. The dynamics of the near-wall boundary layer is found to be governed by a nonlinear equation of motion, which accounts for such mechanisms on surface chains as convection, retraction, constraint release, and thermal fluctuations. This equation is valid over a wide range of grafting regimes, including those in which interactions between neighboring adsorbed molecules become essential. It is not closed since the dynamics of adsorbed chains is shown to be coupled to that of polymer chains in the bulk via constraint release. The constitutive equations for the layer and bulk, together with continuity of stress and velocity, are found to form a closed system of equations which governs the dynamics of the whole "bulk + boundary layer" ensemble. Its solution provides a stick-slip law in terms of the molecular parameters and extruder geometry. The model is quantitative and contains only those parameters that can be measured directly, or extracted from independent rheological measurements. The model predictions show a good agreement with available experimental data. (C) 2005 American Institute of Physics. [DOI: 10.1063/1.1915327]
\end{abstract}

\section{INTRODUCTION}

Wall slip in polymer melts has been a subject of intensive research for the past decades and has been recently reviewed by several authors. ${ }^{1-3}$ Two different mechanisms were proposed to explain the origin of wall slip. The first view was conceptualized by Bergem. ${ }^{4}$ It suggests that wall slip stems from a sudden disentanglement of surface polymer chains adsorbed on the wall from those in the polymer bulk at a critical stress. Slip due to disentanglement occurs in the vicinity of the wall, and is often referred to as cohesive slip. The second view explains slip by adhesive failure of polymer chains at the surface. This type of slip is usually referred to as adhesive slip. It is likely that in reality both slip mechanisms occur simultaneously, and therefore must be taken into account self-consistently. However, it is generally believed that on a high surface energy wall slip mainly occurs due to disentanglement.

The theoretical foundation for wall slip due to disentanglement was developed by Brochard-Wyart and de Gennes ${ }^{5}$ who proposed a scaling model for a flow of a polymer melt over a wall on which chains of the same polymer are adsorbed. The model was developed for the case of low grafting density, in which neighboring surface chains do not overlap. The surface chains are only entangled with the flowing bulk ones. Brochard-Wyart and de Gennes argued that at

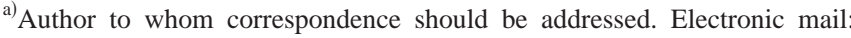
m.tchesnokov@utwente.nl

${ }^{b)}$ Also at Department of Mathematics and Computing Science, Eindhoven University of Technology, P.O. Box 513, 5600 MB Eindhoven, Netherlands.

c) Also at Material Science Centre, DSM Research, P.O. Box 18, 6160 MD Geleen, Netherlands.
}

low flow rates each surface chain can be considered as a random walk, and thus has a coil-like shape. However, a fast flow may deform the chain into a "cigar"-shaped coil. When the diameter of the deformed cylindrical coil decreases below the entanglement spacing, bulk chains suddenly disentangle from surface ones and slip occurs. So the BrochardWyart-de Gennes model relates the stick-slip transition near the wall to a "coil-to-cigar" transition of surface chains. Similar scaling theories for wall slip have been developed by Ajdari et al. ${ }^{6}$ and Mhetar and Archer. ${ }^{7}$ Recently, Joshi et $a l^{8,9}$ proposed a molecular model for cohesive slip, based on the microscopic consideration of the boundary layer and processes on surface chains. The surface chains were shown to undergo a "suppressed" convective constraint release, whose strength determines the resistibility of the boundary layer to the flow. It is argued that above a critical flow rate suppressed convective constraint release is no longer able to "randomize" the orientation of surface chains (caused by the flowing bulk) which leads to a sudden disentanglement between bulk and surface chains. Apart from disentanglement models, several adhesive-failure theories have been proposed in which the role of stress is usually to alter the activation energy for detachment (see, for example, Hill ${ }^{10}$ and Hatzikiriakos ${ }^{11}$ ).

The goal of the present paper is to develop a quantitative molecular model for cohesive slip. Since disentanglement is more likely to be the dominant slip mechanism on a high surface energy wall, where high grafting densities of surface chains are expected, this model should be able to account for interactions between neighboring surface molecules. Our approach differs from earlier slip models. Its basis was partially 


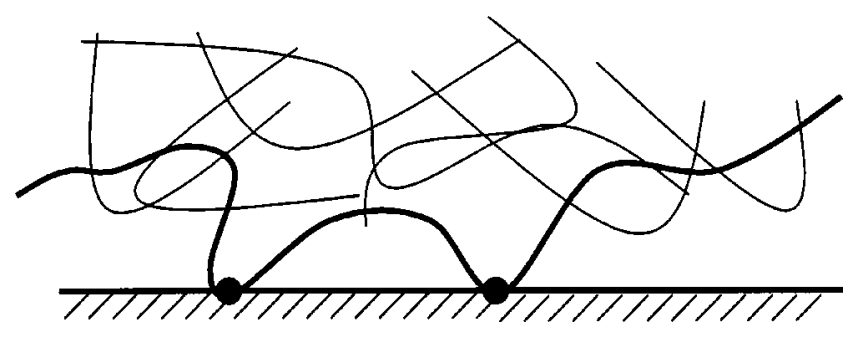

FIG. 1. The near-wall layer. The thin and thick lines stand for bulk and surface chains, respectively.

developed in our previous work, ${ }^{12}$ in which the dynamics of surface chains was studied, and a constitutive equation for the wall stress was derived.

\section{THE INTERFACIAL LAYER}

In a real extrusion system, a molten polymer comes into contact with the solid wall of a die. Due to the presence of attractive polymer-wall interaction, some of the polymer molecules in the near-wall layer become adsorbed on the wall surface (see Fig. 1). In general, each of them makes several connections with the wall, thereby forming so-called loops and tails. The loop is a part of an adsorbed molecule between adjacent polymer-wall connection points. Its both ends are attached to the wall. Being highly restricted in motion, in the presence of flow, loops will be most likely squeezed against the wall by the moving mesh of surrounding chains, and therefore will not interact with the flowing bulk. In this paper, we will ignore the presence of loops, thus assuming that each adsorbed molecule can only interact with the bulk via its tails. The tail is a part of an adsorbed molecule that has only one connection with the wall. It is much more mobile than the loop, and can renew its spatial configuration via constraint release (CR), retraction, or thermal fluctuations. Notice that every surface chain has two tails. Although they belong to the same molecule, their motions can be considered as being independent of each other. In the following, each tail will be regarded as a separate tethered chain. In general, all tethered chains present in the layer have different numbers of monomers and consequently different lengths. However, as a first approximation, we will assume that at equilibrium every tethered chain has the same length equal to $L_{I 0}$, the average equilibrium length of tethered chains.

The average number of connections between the wall and a surface molecule depends on the strength of the polymer-wall interaction. On a high-energy wall, the adsorbed chain can make many connections with the wall so that $L_{I 0}$ is much smaller than $L_{B 0}$, the equilibrium length of a bulk molecule. This in turn implies that the thickness of the near-wall layer which contains surface chains is also much smaller than $L_{B 0}$. Note that this layer separates the flowing bulk and the wall, and therefore will be referred to as the interfacial layer throughout the paper.

In the picture of disentanglement mechanics proposed by Bergem $^{4}$ and afterwards used in scaling and molecular models on slip, it is the dynamics of surface chains that governs the stick-slip transition. On a microscopic level, as noted by
Joshi et al., ${ }^{8}$ the dynamics of tethered chains is determined by the following processes: convection, retraction, constraint release, and thermal fluctuations. Convection, i.e., deformation of the primitive path of a tethered chain by the flowing bulk, rotates and stretches the tethered chain. It tends to align the chain with the flow direction, and will ultimately squeeze it against the wall. Due to convection, tethered chains are stretched in the presence of flow. Hence, the motion of a tethered chain due to convection is always accompanied by its retraction inside the tube, which works in parallel and independently of convection. The tube in which a tethered chain is confined is built out of the surrounding chains. Motion of one of them may result in a release of a constraint on the tethered chain. After this, according to Marrucci, ${ }^{13}$ the tethered chain may experience a random local jump over a distance of the order of the tube diameter, which results in a local relaxation of the tethered chain conformation. So, constraint release randomizes configurations of tethered chains, thus preventing their alignment by convection. Its strength actually measures the resistibility of the interfacial layer to the flow. Finally, the tethered chain also undergoes thermal motion inside its tube which leads to fluctuations in the length of its primitive path. These fluctuations are usually referred to as contour length fluctuations, and lead to additional relaxation of the tube near the free end.

On a macroscopic level, as shown in Ref. 12, the ensemble-averaged dynamics of tethered chains can be described in terms of the so-called bond vector probability distribution function (BVPDF) of tethered chains $f^{I}\left(\mathbf{b}, s_{0}, t\right)$, i.e., the probability for a tethered chain to have a bond vector at position $s_{0}$ and time $t$ equal to $\mathbf{b}$. In this description, the primitive path of a tethered chain is represented as a parametric curve where the parameter $s_{0}$ is the equilibrium arclength of a physical chain segment along the chain contour from the tethered end. The actual arclength of the segment is given by the integral $\int_{0}^{s_{0}} d x \lambda(x, t)$, where $\lambda(x, t)$ is the corresponding local stretch of the primitive path. Since $L_{I 0}$ is the length of a tethered chain, its attached and free ends correspond to $s_{0}=0$ and $s_{0}=L_{I 0}$, respectively. The bond vector $\mathbf{b}\left(s_{0}, t\right)$ is defined as the product $\lambda\left(s_{0}, t\right) \mathbf{u}\left(s_{0}, t\right)$, where $\lambda\left(s_{0}, t\right)$ and $\mathbf{u}\left(s_{0}, t\right)$ are the local stretch and unit tangent vector to the primitive path at $s_{0}$ and time $t$, respectively. Clearly, $\mathbf{b}\left(s_{0}, t\right)$ contains information on both local chain stretch and local chain orientation, so $f^{I}\left(\mathbf{b}, s_{0}, t\right)$ is a natural extension of the orientation distribution function of inextensible (i.e., $\lambda \equiv 1)$ chains $\varphi\left(\mathbf{u}, s_{0}, t\right)$ of Doi and Edwards. ${ }^{14}$ The derived equation of motion for $f^{I}\left(\mathbf{b}, s_{0}, t\right)$ (Ref. 12) accounts for convection, retraction, and constraint release. In the case of inextensible chains it boils down to the original equation of motion for $\varphi\left(\mathbf{u}, s_{0}, t\right)$ found by Doi and Edwards ${ }^{14}$ (in the absence of reptation and constraint release). Let us show now that given $f^{I}\left(\mathbf{b}, s_{0}, t\right)$ one can directly calculate the local stress in the interfacial layer $\sigma_{\alpha \beta}^{I}$. According to Doi and Edwards, ${ }^{14} \sigma_{\alpha \beta}^{I}$ can be written as

$$
\sigma_{\alpha \beta}^{I}(t)=\frac{G_{I 0}}{L_{I 0}} \int_{0}^{L_{I 0}} d s_{0} S_{\alpha \beta}^{I}\left(s_{0}, t\right),
$$

where $G_{I 0}$ and $L_{I 0}$ are the elastic modulus of the layer and the equilibrium length of a tethered chain, respectively. Note that 
Eq. (1) does not take into account a contribution of monomer-monomer friction between bulk and tethered chains to the local stress in the layer, which can be neglected in the presence of bulk-tethered entanglements. The tensor $S_{\alpha \beta}^{I}=\left\langle b_{\alpha}\left(s_{0}, t\right) b_{\beta}\left(s_{0}, t\right)\right\rangle_{I}$ is the bond vector correlator where $b_{\alpha}$ is the $\alpha$ component of the bond vector $\mathbf{b}$ of a tethered chain. The brackets $\langle\cdots\rangle_{I}$ denote averaging over the ensemble of tethered chains. Note that this averaging is equivalent to that via the BVPDF, and so

$$
S_{\alpha \beta}^{I}\left(s_{0}, t\right)=\int_{\mathbb{R}^{3}} d^{3} \mathbf{b} b_{\alpha} b_{\beta} f^{I}\left(\mathbf{b}, s_{0}, t\right) .
$$

Equation (2) shows that $S_{\alpha \beta}^{I}$ is the second moment of the BVPDF. Knowing the equation of motion for $f^{I}$, one can directly find that for $S_{\alpha \beta}^{I}$. As found in Ref. 12, in the case of small chain stretch it has the form

$$
\begin{aligned}
\frac{\partial S_{\alpha \beta}^{I}}{\partial t}= & K_{\alpha \gamma}^{I} S_{\beta \gamma}^{I}+K_{\beta \gamma}^{I} S_{\alpha \gamma}^{I}+\frac{3 \nu_{I} a_{0}^{2}}{2} \frac{\partial^{2} S_{\alpha \beta}^{I}}{\partial s_{0}^{2}}+2 \bar{\xi}_{I} S_{\alpha \beta}^{I} \\
& +\int_{0}^{s_{0}} d x \bar{\xi}_{I}(x, t) \frac{\partial S_{\alpha \beta}^{I}}{\partial s_{0}} .
\end{aligned}
$$

The first two terms on the right-hand side (RHS) pertain to convection. The gradient velocity tensor $K_{\alpha \beta}^{I}$ (Ref. 14) gives the average deformation rate of the primitive path of a tethered chain due to convection. Note that in a simple shear flow $K_{\alpha \beta}^{I}$ has only one nonzero component equal to the wall shear rate $\dot{\gamma}_{\omega}$. The third term on the RHS pertains to constraint release. It has the form of a diffusion process with the coefficient proportional to the frequency of constraint release $\nu_{I}$ and the equilibrium tube diameter $a_{0}$ squared. The last two terms on the RHS of Eq. (3) stem from retraction with $\bar{\xi}_{I}\left(s_{0}, t\right)$ being the average retraction rate of the primitive path at position $s_{0}$ and time $t$. If $T_{I R}$ is the characteristic relaxation time of chain stretch due to retraction, then $\bar{\xi}_{I}\left(s_{0}, t\right)$ can be approximated by

$$
\bar{\xi}_{I}\left(s_{0}, t\right) \approx-\frac{\bar{\lambda}_{I}\left(s_{0}, t\right)-1}{T_{I R}}
$$

where $\bar{\lambda}_{I}\left(s_{0}, t\right)$ is the ensemble-averaged local stretch of tethered chains at $s_{0}$ and time $t$. Since the motion of a tethered chain inside its tube can be interpreted as one-dimensional Rouse motion, $T_{I R}$ is equal to the corresponding Rouse time of tethered chains. ${ }^{14}$ The Rouse time $T_{I R}$ of tethered chains is two times larger than the corresponding Rouse time of bulk chains with the same number of monomers. Note that the single relaxation-time approximation in Eq. (4) may not be applicable for segments near the free end where fast equilibration processes are expected to be active. In Eq. (4), $\bar{\lambda}_{I}\left(s_{0}, t\right)$ can be in turn expressed via $S_{\alpha \beta}^{I}$. Since $b_{\alpha}=\lambda u_{\alpha}$ and $\mathbf{u}$ is a unit vector, then

$$
\bar{\lambda}_{I}\left(s_{0}, t\right) \approx \sqrt{\left\langle b_{\alpha} b_{\alpha}\right\rangle_{I}}=\sqrt{S_{\alpha \alpha}^{I}},
$$

where summation is implied over repeated indices. Equation (5) reveals that the equation of motion for the correlator $S_{\alpha \beta}^{I}$ is nonlinear. To solve it, we must also specify the boundary conditions for $S_{\alpha \beta}^{I}$. Since the unattached end of a tethered chain is "free to choose" its direction and all the directions are equally probable, $f^{I}\left(\mathbf{b}, s_{0}, t\right)$ at $s_{0}=L_{I 0}$ is isotropic. The relaxation time of chain segments close to the free end is of the order of $\tau_{e}$, the Rouse time of one segment. So, on the time scale of $T_{I R}$, fast retraction near the free end can be considered instantaneous so that the corresponding chain stretch can be neglected. On the other hand, basing on symmetry arguments, $S_{\alpha \beta}^{I}\left(s_{0}, t\right)$ can be thought of as being an even function of $s_{0}$. Therefore,

$$
S_{\alpha \beta}^{I}\left(s_{0}=L_{I 0}, t\right)=\frac{1}{3} \delta_{\alpha \beta},\left.\quad \frac{\partial S_{\alpha \beta}^{I}\left(s_{0}, t\right)}{\partial s_{0}}\right|_{s_{0}=0}=0 .
$$

Equation (6) shows that the mean local chain stretch at the attached end of a tethered chain is maximal.

In Eq. (3), the contribution of thermal fluctuations, i.e., another possible relaxation mechanism, has not been included yet. These fluctuations involve motion of the free end of a tethered chain inside its tube, thereby temporarily creating a higher than the average density of monomers near the chain end. When the chain end moves outward again, it is free to choose its direction and the initial orientation relaxes. According to Milner and McLeish, ${ }^{15}$ the mean relaxation time of chain segments due to fluctuations increases exponentially with the distance from the chain end and can be written as

$$
\tau_{I}\left(s_{0}\right) \approx \tau_{I 0} e^{1.5 Z_{T}\left(1-s_{0} / L_{I 0}\right)^{2}}
$$

where $Z_{T}$ is the mean number of constraints per tethered chain. In Eq. (7) use was made of the fact that chain stretch is small near the free end of a tethered chain. For the time constant $\tau_{I 0}$ we use the Rouse time of a tethered chain $T_{I R}$. A more accurate prefactor, which depends on $s_{0}$, was derived in Ref. 15. In view of Eq. (7), the contribution of thermal fluctuations into the equation of motion for $S_{\alpha \beta}^{I}$ [Eq. (3)] has the form

$$
-\frac{1}{\tau_{I}\left(s_{0}\right)}\left[S_{\alpha \beta}^{I}\left(s_{0}, t\right)-S_{\alpha \beta}^{\mathrm{eq}}\right], \quad S_{\alpha \beta}^{\mathrm{eq}}=\frac{1}{3} \delta_{\alpha \beta},
$$

where $S_{\alpha \beta}^{\mathrm{eq}}$ is the equilibrium correlator corresponding to the isotropic BVPDF and $\lambda \equiv 1$. Equation (7) explicitly shows that thermal fluctuations become especially important for short (i.e., small $Z_{T}$ ) tethered chains.

According to Eq. (1), the wall stress is proportional to the averaged along the tethered chain contour value of the correlator $S_{\alpha \beta}^{I}$, so Eq. (3) extended with Eq. (8) actually forms the constitutive equation for the interfacial layer. A layer constitutive equation was also derived by Joshi et al. ${ }^{8}$ who extended the contour variable of Mead et al. ${ }^{16}$ to the case of tethered chains. Their model is formulated in terms of the tube survival probability $G\left(s_{0}, t\right),{ }^{14}$ whereas in our approach the equation for the stress is written directly. Note that we have not yet explicitly specified $\nu_{I}$, the frequency of constraint release on tethered chains. In order to complete the obtained constitutive equation, in Sec. III we will study constraint release on tethered chains and find an explicit expression for $\nu_{I}$. 


\section{CONSTRAINT RELEASE IN THE INTERFACIAL LAYER}

In general, every tethered chain is entangled with other tethered and bulk chains present in the layer (see Fig. 1). This implies two sorts of constraints on a tethered chain. Those of the first sort are imposed by bulk chains, and can be released via their reptation (thermal constraint release) or retraction (convective constraint release). Constraints of the second sort are imposed by other tethered chains and released via thermal fluctuations of their free ends (the socalled arm retraction). According to Ajdari and co-workers, ${ }^{6,17}$ the characteristic time scale of arm retraction $\tau_{\mathrm{AR}}$, that is to say the mean lifetime of a "tethered" constraint, is given by

$$
\tau_{\mathrm{AR}} \approx \tau_{d}\left(Z_{T}\right) Z_{T}^{-1} \exp \left(\alpha Z_{T}\right),
$$

where $\tau_{d}\left(Z_{T}\right)$ is the reptation time ${ }^{14}$ of a free chain which has $Z_{T}$ entanglement segments, and $\alpha=15 / 10$ is a numerical prefactor. The characteristic time scale of thermal constraint releases $\tau_{\mathrm{TCR}}$, i.e., the mean time necessary to remove one "bulk" constraint via reptation, was calculated earlier in Ref. 18 [see equation below Eq. (21)]. As found in Ref. 18, $\tau_{\mathrm{TCR}}$ can be estimated as

$$
\tau_{\mathrm{TCR}} \approx \tau_{d}\left(Z_{B}\right) Z_{B}^{-1} \text {. }
$$

This equation shows that for $Z_{B}<100, \tau_{\mathrm{AR}}$ is of the order of $\tau_{\mathrm{TCR}}$ only for very short tethered chains with $Z_{T}<5$. For tethered chains with $Z_{T}>5$, the lifetime of a tethered constraint is much larger than that of a bulk one, so it is possible to think that only bulk constraints can be released. As mentioned before, the thickness of the interfacial layer is usually much smaller than the length of bulk molecules. This means that the bulk chains present in the layer are actually short fragments of molecules from the polymer bulk. Therefore, the frequency of constraint release on tethered chains $\nu_{I}$ can be written as

$$
\nu_{I}=\nu_{B} \phi_{Z},
$$

where $\nu_{B}$ is the corresponding frequency of constraint release in the bulk, and $\phi_{Z}$ is the fraction of bulk constraints per tethered chain. A similar relation between $\nu_{I}$ and $\nu_{B}$ was derived in Ref. 9. Note that $\nu_{B}$ is inversely proportional to the mean lifetime of a constraint in the bulk, and thus is a function of the bulk flow rate. To calculate $\phi_{Z}$, let us assume that all the entanglements in the layer are pairwise contacts between separate polymer chains. Then, the entanglement network in the layer can be imagined as being built out of interacting "half-entanglements" (see Fig. 2).

Every tethered chain contributes to $Z_{T} / 2$ entanglements, or equivalently provides $Z_{T}$ tethered half-entanglements. Every bulk chain present in the layer may provide $u p$ to $Z_{B}$ bulk half-entanglements, where $Z_{B}$ is the mean number of constraints per molecule in the bulk. A half-entanglement "interacts" with another tethered or bulk one available in the layer. Two half-entanglements build an entanglement of one of the three types: tethered-tethered (T-T), bulk-bulk (B-B), or tethered-bulk (B-T). Let $W_{T}$ and $W_{B}$ be the fractions of tethered and bulk half-entanglements per unit volume in the in-

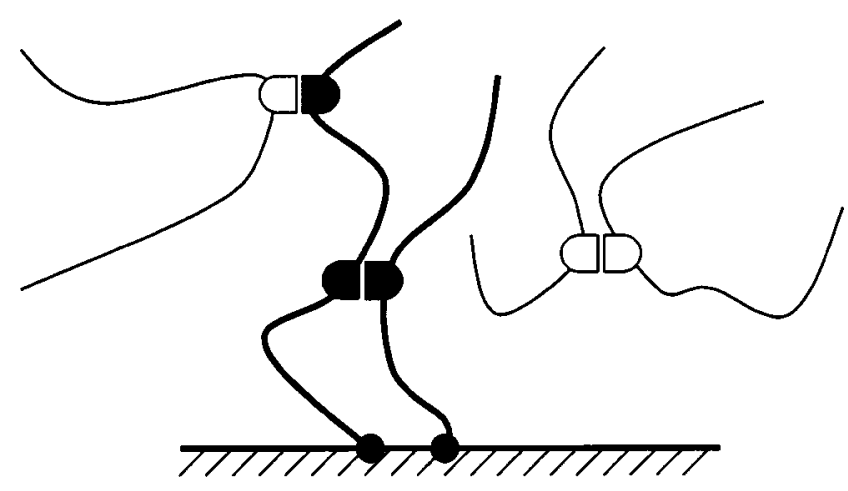

FIG. 2. The entanglement network in the interfacial layer.

terfacial layer, respectively. Since half-entanglements are distributed homogeneously in the layer, corresponding fractions of entanglements of each type per unit volume in the layer are given by

$$
W_{B B}=W_{B} W_{B}, \quad W_{T T}=W_{T} W_{T}, \quad W_{B T}=2 W_{B} W_{T} .
$$

The factor 2 in the expression for $W_{B T}$ stems from $W_{B T}$ $=W_{T B}$. Let $\Sigma_{T}$ be the surface density of tethered chains, that is the number of tethered chains per unit area on the wall. The number of surface molecules adsorbed on the wall is then equal to $\Sigma_{T} / 2$. Since each tethered chain provides $Z_{T}$ tethered half-entanglements, the number of tethered halfentanglements per unit area of the wall in the layer is given by $Z_{T} \Sigma_{T}$. On the other hand, the corresponding total number of half-entanglements per unit area is

$$
\frac{2 h}{a_{0}^{3}}
$$

where $h$ and $a_{0}$ are the thickness of the interfacial layer and mean distance between entanglements, respectively. $a_{0}$ represents the mesh size of the entanglement network in the melt. It is of the same order as the tube diameter and step length (see also Doi and Edwards ${ }^{14}$ ).

In Eq. (12), $a_{0}^{3}$ gives the volume "occupied" by a single entanglement. The volume fractions of bulk and tethered half-entanglements in the layer are then given by

$$
W_{T}=a_{0}^{3} \frac{Z_{T} \Sigma_{T}}{2 h}, \quad W_{B}=1-a_{0}^{3} \frac{Z_{T} \Sigma_{T}}{2 h},
$$

where use was made of the fact that $W_{T}+W_{B}=1$. If the surface density $\Sigma_{T}$ of tethered chains is equal to the critical value,

$$
\Sigma_{T}^{*}=\frac{2 h}{a_{0}^{3}} \frac{1}{Z_{T}}
$$

the volume fraction of bulk half-entanglements $W_{B}$ in the layer vanishes, which means that the layer no longer contains bulk chains. At high surface densities $\Sigma_{T} \geqslant \Sigma_{T}^{*}$, bulk chains are "expelled" from the layer, so it is only populated by tethered ones. This regime is usually referred to as the drybrush regime. ${ }^{9}$ With the help of $\Sigma_{T}^{*}$ [Eq. (14)], Eq. (13) reads as 


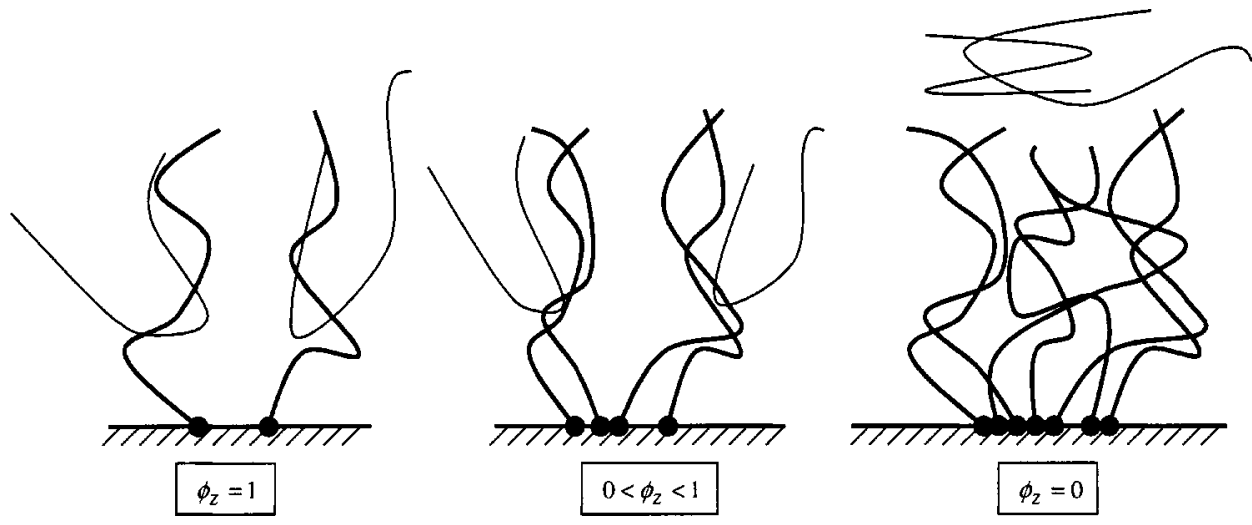

FIG. 3. Grafting regimes. From left to right: mushroom regime, intermediate regime, dry-brush regime. The thick and thin lines stand for tethered and bulk molecules, respectively.

$$
W_{T}=\frac{\Sigma_{T}}{\Sigma_{T}^{*}}, \quad W_{B}=1-\frac{\Sigma_{T}}{\Sigma_{T}^{*}} .
$$

In terms of $W_{\mathrm{B}}$ and $W_{\mathrm{T}}$, the mean fraction of bulk constraints per tethered chain $\phi_{Z}$ is given by

$$
\phi_{Z}=\frac{W_{B T}}{W_{T T}+W_{B T}}=\frac{2 W_{B}}{W_{T}+2 W_{B}},
$$

where use was made of Eq. (11). Clearly, in the dry-brush regime, $\phi_{Z}=0$, so a tethered chain has only tethered constraints, as expected. In the opposite case of very low surface densities, entanglements between neighboring tethered chains are unlikely, so that all the constraints on a tethered chain are bulk, and $\phi_{Z}=1$. This regime is often referred to as the mushroom regime. There is an intermediate grafting regime in which every tethered chain has both bulk and tethered constraints, and so $\phi_{Z}<1$ (see Fig. 3).

A typical behavior of the fraction $\phi_{Z}$ versus the surface density $\Sigma_{T}$ is shown in Fig. 4. It is seen that $\phi_{Z}$ is a monotonously decreasing function of $\Sigma_{T}$ which implies that the role of interactions between neighboring tethered chains increases with $\Sigma_{T}$. At low $\Sigma_{T}$, where tethered chains do not

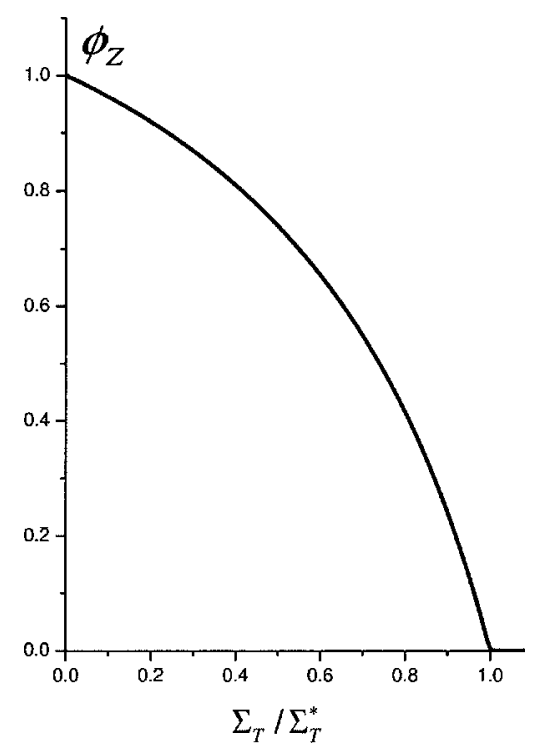

FIG. 4. Fraction of 'bulk' constraints per tethered chain vs surface density of tethered chains. overlap, $\phi_{Z} \approx 1$, and the frequency $\nu_{I}$ is equal to that in the polymer bulk $\nu_{B}$. However, at higher $\Sigma_{T}$ where interactions between tethered chains become essential, $\nu_{I}$ can be much smaller than $\nu_{B}$. In this regime constraint release on tethered chains is "suppressed" by tethered-tethered chain interactions. As mentioned before, it is constraint release which prevents the alignment of tethered chains by the flow and determines the resistibility of the interfacial layer to the flow. By reducing $\nu_{I}$, we "ease" the alignment, thereby facilitating slip. Therefore, at high grafting densities suppressed constraint release on tethered chains may lead to the onset of slip even at rather small flow rates.

Equation (10) shows that the frequency of constraint release $\nu_{I}$ on tethered chains depends on the bulk flow rate (via $\nu_{B}$ ) and the surface energy of the wall (via $\Sigma_{T}$ ). It may also depend on the flow rate in the layer via the thickness $h$ [see Eq. (14)]. The dependence of $\nu_{I}$ on the parameters of the bulk implies that the dynamics of tethered chains (and accordingly interfacial layer) is coupled to that of polymer chains in the bulk. As a consequence, in order to quantify the stick-slip transition, we must consider the dynamics of the whole, i.e., "bulk+interfacial layer," system. To this end, in Sec. IV we will derive a constitutive equation for bulk chains in the presence of flow.

\section{BULK REGION}

Similar to the wall stress, the local stress in the bulk can be presented as

$$
\sigma_{\alpha \beta}^{B}(t)=\frac{G_{B 0}}{L_{B 0}} \int_{-L_{B 0} / 2}^{L_{B 0} / 2} d s_{0} S_{\alpha \beta}^{B}\left(s_{0}, t\right),
$$

where $L_{B 0}, S_{\alpha \beta}^{B}$, and $G_{B 0}$ are the equilibrium length of bulk chains, the bond vector correlator, and the elastic modulus of the bulk, respectively. In the case of bulk chains it is convenient to choose the interval for the parameter $s_{0}$ as $-L_{B 0} / 2$ $<s_{0}<L_{B 0} / 2$, where segments with $s_{0}= \pm L_{B 0} / 2$ correspond to the free ends of a chain. Bulk chains undergo the same mechanisms as tethered ones and may also "reptate." So the equation of motion for $S_{\alpha \beta}^{B}$ can be readily inferred from Eq. (3) if we also take into account reptation. According to Doi and Edwards, ${ }^{14}$ the contribution of reptation to the equation of motion for $S_{\alpha \beta}^{B}$ of inextensible chains has the form of a 
diffusion process with coefficient $D_{c}$, the diffusion coefficient of reptation. Note that reptation is only important in the absence of flow or in slow flow, that is when the polymer chains are not stretched. Moreover, reptation is independent of other mechanisms on bulk chains. Therefore, from Eq. (3) we immediately have (for certainty $0<s_{0}<L_{B 0} / 2$ )

$$
\begin{aligned}
\frac{\partial S_{\alpha \beta}^{B}}{\partial t}= & K_{\alpha \gamma}^{B} S_{\beta \gamma}^{B}+K_{\beta \gamma}^{B} S_{\alpha \gamma}^{B}+\left(D_{c}+\frac{3 \nu_{B} a_{0}^{2}}{2}\right) \frac{\partial^{2} S_{\alpha \beta}^{B}}{\partial s_{0}^{2}} \\
& +2 \bar{\xi}_{B} S_{\alpha \beta}^{B}+\int_{0}^{s_{0}} d x \bar{\xi}_{B}(x, t) \frac{\partial S_{\alpha \beta}^{B}}{\partial_{s_{0}}}-\frac{1}{\tau_{B}}\left(S_{\alpha \beta}^{B}-S_{\alpha \beta}^{\mathrm{eq}}\right),
\end{aligned}
$$

where $S_{\alpha \beta}^{\mathrm{eq}}$ is the equilibrium value of the bond vector correlator defined in Eq. (8). Here, $K_{\alpha \beta}^{B}$ is the bulk gradient velocity tensor. In a simple shear flow, $K_{\alpha \beta}^{B}$ has only one nonzero component equal to the bulk shear rate $\dot{\gamma}_{b}$. The corresponding mean lifetime $\tau_{B}\left(s_{0}\right)$ of the tube segment $s_{0}$ of a bulk chain due to thermal fluctuations is given by

$$
\tau_{B}\left(s_{0}\right) \approx T_{B R} e^{0.75 Z_{B}\left(1-2 s_{0} / L_{B 0}\right)^{2}},
$$

where $T_{B R}$ is the Rouse time of bulk chains. In Eq. (18), $\bar{\xi}_{B}\left(s_{0}, t\right)$ is the corresponding retraction rate of the primitive path at $s_{0}$ and time $t$. In the single relaxation-time approximation $\bar{\xi}_{B}\left(s_{0}, t\right)$ reads as

$$
\bar{\xi}_{B}\left(s_{0}, t\right) \approx-\frac{\bar{\lambda}_{B}\left(s_{0}, t\right)-1}{T_{B R}},
$$

where $\bar{\lambda}_{B}$ is the mean local stretch of bulk chains. In order to "close" Eq. (18) and complete the model, we must specify an explicit expression for $\nu_{B}$, the frequency of constraint release in the bulk. In general, constraints on bulk chains are released by reptation [thermal constraint release (TCR)] or retraction [convective constraint release (CCR)] of surrounding molecules. The frequency of CCR can be readily found if we assume that retraction of one free end of a bulk molecule over a distance equal to the mean entanglement spacing $a_{0}$ releases one constraint on another chain. Basing on this observation, in Ref. 18 we found that the frequency $\nu_{B}$ of CCR on bulk chains is given by

$$
\nu_{B}=2 \frac{2}{a_{0} Z_{B}}\left[\int_{0}^{L_{0} / 2} d x \mid \bar{\xi}_{B}(x, t)\right] .
$$

The expression in square brackets is the retraction rate of a chain end inside the tube, i.e., the average velocity between the free end and the tube. Equation (21) also accounts for the fact that only a fraction of $2 / Z_{B}$ of the constraints will on average be released on a chain during the characteristic time interval needed for bulk chains to retract its end over the distance $a_{0}$. The prefactor 2 on the RHS indicates that both ends of a chain contribute to CCR. Basing on similar arguments, the frequency of TCR was found to be

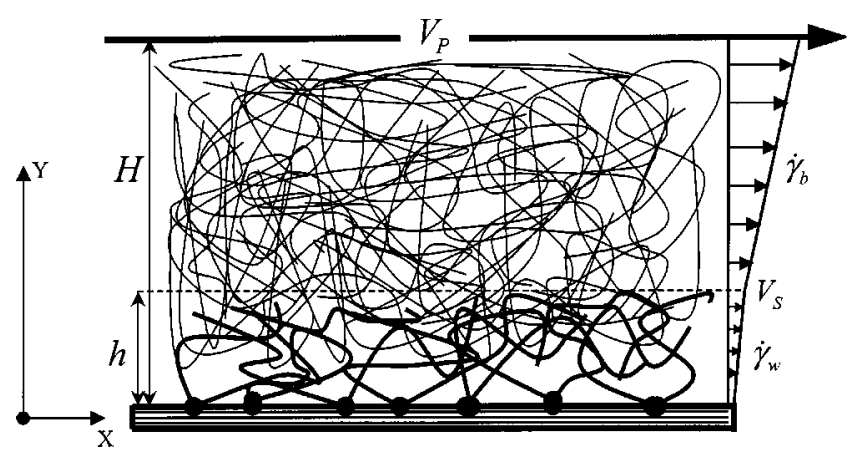

FIG. 5. The parallel-plate geometry: $H$ is the distance between plates, $h$ is the thickness of the interfacial layer, and $\dot{\gamma}_{w}$ and $\dot{\gamma}_{b}$ are the shear rates in the layer and the bulk, respectively. The slip velocity $V_{s}$ is defined as the average velocity of monomers at the top of the layer.

$$
\nu_{B}=\frac{4 D_{c}}{Z_{B} \alpha_{0}^{2}} .
$$

The derived equation of motion for the correlator $S_{\alpha \beta}^{B}$ describes the time evolution of the average configuration of bulk chains in flow. One may ascertain that it can also be derived from the corresponding equation of motion for the BVPDF of bulk chains found in Ref. 18. Note that Eq. (18) can be written in the form of the Rolie-Poly bulk constitutive equation proposed by Likhtman and Graham ${ }^{19}$ if one neglects high order modes due to CCR and retraction, as well as the position dependence of the local chain stretch. In Sec. $\mathrm{V}$ a molecular model for slip will be formulated which combines both the equation of motion for the interfacial layer and for the bulk.

\section{MODEL FOR SLIP AT POLYMER/SOLID INTERFACE}

In this section, a molecular model for wall slip is formulated for the case of parallel-plate geometry for which precise experimental data on slip are available. A simple (not on scale) sketch of a parallel-plate rheometer is shown in Fig. 5. It is a controlled shear rate apparatus in which a homogeneous polymer melt is confined between two metal plates. The lower plate is fixed and will be referred to as 'wall' throughout the text. The upper plate is moving at a certain constant velocity $V_{p}$. No slippage is assumed between the upper plate and the melt. The movement of the upper plate builds up a linear velocity profile in the melt. However, as seen in Fig. 5, there are two different flow regions that must be distinguished. The first one is the bulk. It only contains bulk polymer chains and has the shear rate $\dot{\gamma}_{b}$. The second flow region is the interfacial layer. It contains both surface and bulk chains and has the shear rate $\dot{\gamma}_{w}$.

Our goal is to describe quantitatively the stick-slip transition in such a system. A successful stick-slip law must be able to predict the dependence of slip parameters of the system (such as the slip velocity) as well as the disentanglement point on the molecular data and processing conditions. In the previous sections we considered the dynamics of tethered chains, which is believed to be the "clue" to the onset of slip. As discussed above, it is coupled to the dynamics of polymer molecules in the bulk. To derive a quantitative stick-slip law (i.e., the slip velocity $V_{s}$ versus the velocity of the upper 
plate $V_{p}$ ), we have to consider the dynamics of the whole flow, namely, the bulk+interfacial layer. The derived equations of motion for the interfacial layer [see Eq. (3)] and for the bulk [see Eq. (18)] allow to calculate the slip velocity $V_{s}$ provided that the corresponding shear rates $\dot{\gamma}_{b}$ and $\dot{\gamma}_{w}$ are known. However, $\dot{\gamma}_{b}$ and $\dot{\gamma}_{w}$ are not independent parameters, but coupled functions of $V_{p}$. To determine them selfconsistently, we need two extra equations. The first one stems from the continuity of the average velocity of monomers in the melt which can be written as

$$
h \dot{\gamma}_{w}+\dot{\gamma}_{b}(H-h)=V_{p},
$$

where $H$ is the distance between plates. The first term on the left-hand side (LHS) gives the slip velocity $V_{s}$, which is defined as the average velocity of monomers at the top of the layer, whereas the second represents the change in the melt velocity over the bulk. As shown in Ref. 12, the layer thickness $h$ is a nonlinear function of $\dot{\gamma}_{w}$. Besides that, $h$ also depends on $\dot{\gamma}_{b}$ via $\nu_{l}$, the frequency of CR on tethered chains. So, given $\dot{\gamma}_{b}$, Eq. (22) yields a nonlinear equation for $\dot{\gamma}_{w}$. In the molecular model of Joshi et al., ${ }^{8}$ the dependence of the layer thickness $h$ on $\dot{\gamma}_{w}$ was neglected in Eq. (22), and instead its equilibrium value was used. This may result in an overestimate of $V_{s}$ near the transition point where significant alignment of tethered chains by the flow is expected. The second equation relating $\dot{\gamma}_{b}$ and $\dot{\gamma}_{w}$ stems from the continuity of stress at the interface between the bulk and interfacial layer:

$$
\sigma_{x y}^{B}\left(\dot{\gamma}_{b}\right)=\sigma_{x y}^{I}\left(\dot{\gamma}_{w}\right)
$$

As found in Ref. 12, the wall shear stress $\sigma_{x y}^{I}$ is a nonlinear function of $\dot{\gamma}_{w}$. Moreover, it also depends on $\dot{\gamma}_{b}$ via the frequency of CR in the layer. Therefore, Eq. (23) yields another nonlinear relation between $\dot{\gamma}_{b}$ and $\dot{\gamma}_{w}$. To proceed, we also need to incorporate the elastic moduli $G_{B 0}$ and $G_{I 0}$ [see Eqs. (1) and (17)]. Note that to solve Eq. (23) we do not need to know their absolute values. Instead, we only need to specify their ratio $G_{I 0} / G_{B 0}$. Let us show now that this ratio can be expressed in terms of the molecular parameters of the melt and surface density of tethered chains $\Sigma_{T}$. As follows from the rubber elasticity theory (see, for example, de Gennes, ${ }^{20}$ ) the elastic modulus of the bulk $G_{B 0}$ is proportional to the equilibrium number of entanglements per unit volume $1 / a_{0}^{3}$, where $a_{0}$ is the mean distance between entanglements. In order to find the coefficient, let us point out a unit volume in the melt which contains, say $\rho_{n}$ polymer chains. If all the entanglements in the melt are pairwise contacts between separate polymer chains, then the number of entanglements per unit volume is

$$
\frac{\rho_{n}}{2} \bar{Z}=\frac{1}{a_{0}^{3}},
$$

where $\tilde{Z}=M / M_{e}$ is the mean number of constraints per chain. Here $M$ is the mean molecular weight of polymer chains, and $M_{e}$ is the mean molecular weight between entanglements. Therefore, according to Doi and Edwards, ${ }^{14}$ we have that

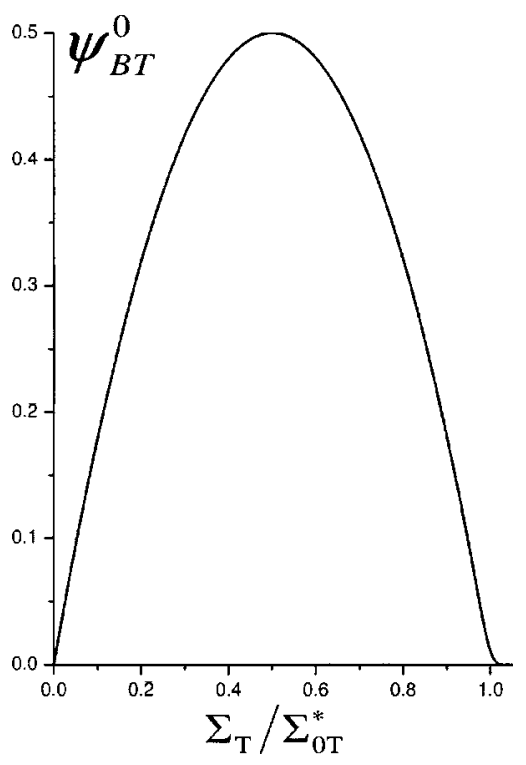

FIG. 6. Equilibrium volume fraction of B-T entanglements in the layer vs surface density. $\Sigma_{0 T}^{*}$ is the equilibrium surface density of the dry-brush regime.

$$
G_{B 0}=\frac{\rho_{n} M k_{B} T}{M_{e}}=\frac{2 k_{B} T}{a_{0}^{3}} .
$$

A similar expression can be written for $G_{I 0}$. However, attention must be paid that it is mainly entanglements between bulk and tethered chains that are active in transferring stress from the flowing bulk to the interfacial layer. Therefore, the elastic modulus of the interfacial layer can be approximated as

$$
G_{I 0} \approx \frac{2 k_{B} T}{a_{0}^{3}} \psi_{B T}^{0}
$$

where $\psi_{B T}^{0}$ is the equilibrium volume fraction of bulktethered $(\mathrm{B}-\mathrm{T})$ entanglements in the layer. Its explicit form can be readily found from the half-entanglements model discussed above. From Eq. (11), $\psi_{B T}^{0}$ is given by

$$
\frac{G_{I 0}}{G_{B 0}}=\psi_{B T}^{0}=\frac{W_{B T}}{W_{B B}+W_{B T}+W_{T T}}=2 W_{B}^{0} W_{T}^{0},
$$

where $W_{B}^{0}$ and $W_{T}^{0}$ are the equilibrium volume fractions of bulk and tethered half-entanglements, respectively. An explicit form of $W_{B}^{0}$ and $W_{T}^{0}$ directly follows from Eq. (13) if the layer thickness $h$ is replaced with its equilibrium value. In the mushroom regime, in which neighboring tethered chains do not overlap, from Eq. (26) it follows that the wall shear stress $\sigma_{x y}^{I}$ is proportional to the surface density of tethered chains $\Sigma_{T}$, similar to the behavior predicted by the slip model of Brochard-Wyart and de Gennes ${ }^{5}$ and molecular model of Joshi et $a l^{8}$ On the other hand, at high grafting densities with $W_{B} \ll W_{T}$, the wall shear stress $\sigma_{x y}^{I}$ is proportional to $\phi_{Z}^{0} \Sigma_{T}$, where $\phi_{Z}^{0}$ is the equilibrium fraction of bulk constraints per tethered chain (see Fig. 4). Note that in the absence of desorption, $\Sigma_{T}$ is a constant independent of the melt temperature and shear rate.

A typical behavior of $\psi_{B T}^{0}$ as a function of $\Sigma_{T}$ is presented in Fig. 6. As is seen, $\psi_{B T}^{0}$ is a nonmonotonous function 


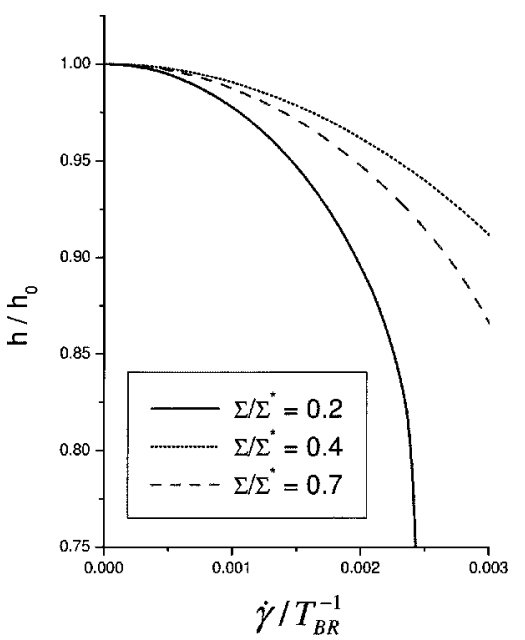

FIG. 7. Layer thickness vs shear rate for different grafting densities, for $Z_{B}=30$ and $Z_{T}=10$.

of $\Sigma_{T}$. At small $\Sigma_{T}, \psi_{B T}^{0}$ increases linearly with $\Sigma_{T}$. In this regime, the number of bulk-tethered $(\mathrm{B}-\mathrm{T})$ entanglements in the layer is small compared to entanglements between bulk chains. Moreover, entanglements between neighboring tethered chains are unlikely, so that different tethered chains contribute independently to $\psi_{B T}^{0}$. Inclusion of one additional tethered chain in the layer will create $Z_{T}$ new B-T entanglements. At a certain critical surface density, which is of the order of the overlap surface density, entanglements between neighboring tethered chains start to play an important role in the entanglement network. In this regime, all bulk chains available in the layer are already "captured" by tethered chains so that inclusion of one new tethered chain will not create new B-T entanglements. Instead, some tetheredtethered entanglements will be created, and as a result some of the bulk constraints on tethered chains will be replaced with tethered ones. By increasing $\Sigma_{T}$, connections between tethered chains become more and more favorable, and so bulk molecules are gradually expelled from the layer. This results in a decrease in the corresponding density of B-T entanglements. At $\Sigma_{T}=\Sigma_{T}^{*}$ the layer enters the dry-brush regime, in which there are no more B-T entanglements in the layer.

By now we have found the constitutive equations for the layer and for the bulk. We also found that continuity of velocity and stress at the bulk-layer interface provide an explicit dependence of the bulk and wall shear rates on the upper plate velocity. Equations (3), (18), (22), and (23) lie at the heart of the model. They form a closed system of equations whose solution provides a detailed information about the dynamics of the melt in terms of the molecular and surface parameters, and the die geometry. In Sec. VI, predictions of the model for the stick-slip law will be presented.

\section{RESULTS AND DISCUSSION}

The final system of Equations (3), (18), (22), and (23) was solved numerically using the conventional Newton method. Steady-state model predictions for the stick-slip law are presented in Figs. 7-15. In Fig. 7, the layer thickness $h$ is shown as a function of the "global" shear rate $\dot{\gamma}=V_{p} / H$ for

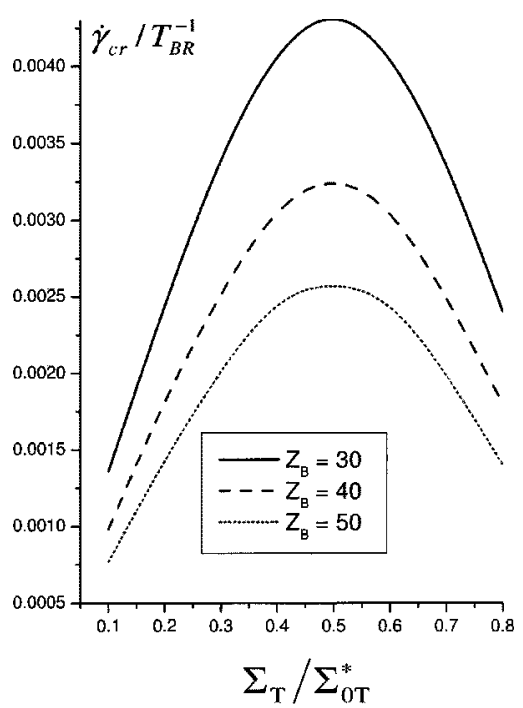

FIG. 8. Critical shear rate vs surface density for different $Z_{B}$, for $Z_{T}=10$.

different surface densities of tethered chains. The thickness $h$ was estimated as the ensemble average $\left\langle\hat{\mathcal{R}}_{y} \hat{\mathcal{R}}_{y}\right\rangle^{-1 / 2}$, where $\hat{\mathcal{R}}_{y}$ was the $y$ component of the end-to-end vector of a tethered chain. As shown in Ref. 18, in the absence of a longrange interaction between chain segments along the contour, this correlator can be expressed via the averaged along the chain contour value of $S_{y y}^{I}$, the $y y$ component of the bond vector correlator of tethered chains [see Eq. (2)]. Therefore,

$$
h^{2} \approx\left\langle\hat{\mathcal{R}}_{y} \hat{\mathcal{R}}_{y}\right\rangle \approx \frac{3 h_{0}^{2}}{L_{0}} \int_{0}^{L_{0}} d s_{0} S_{y y}^{I}\left(s_{0}, t\right),
$$

where the coefficient before the integral makes sure that in the absence of flow $h$ is equal to $h_{0}$, the equilibrium layer thickness. An explicit expression for $h_{0}$ can be easily found from the freely jointed chain model. ${ }^{14}$ As in the absence of flow each tethered chain is a random walk and has a coil-like shape; $h_{0}$ can be estimated as the mean diameter of the coil given by $\sqrt{N_{T}} b$, where $N_{T}$ is the number of monomers per tethered chain, and $b$ is the monomer size.

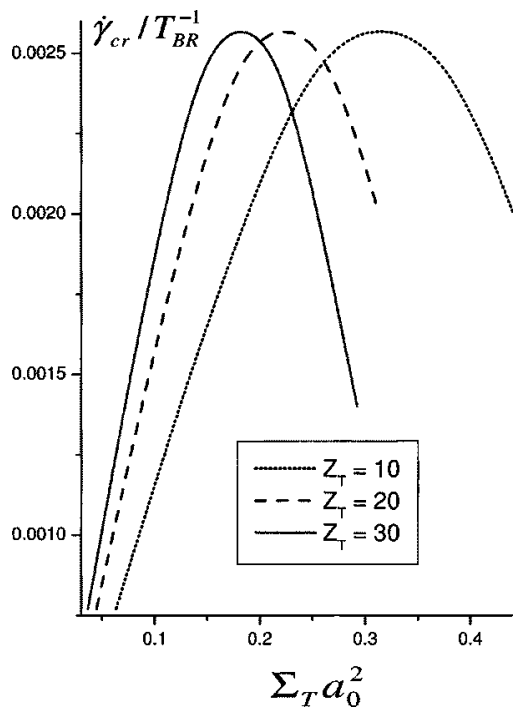

FIG. 9. Critical shear rate vs surface density for different $Z_{T}$, for $Z_{B}=50$. 


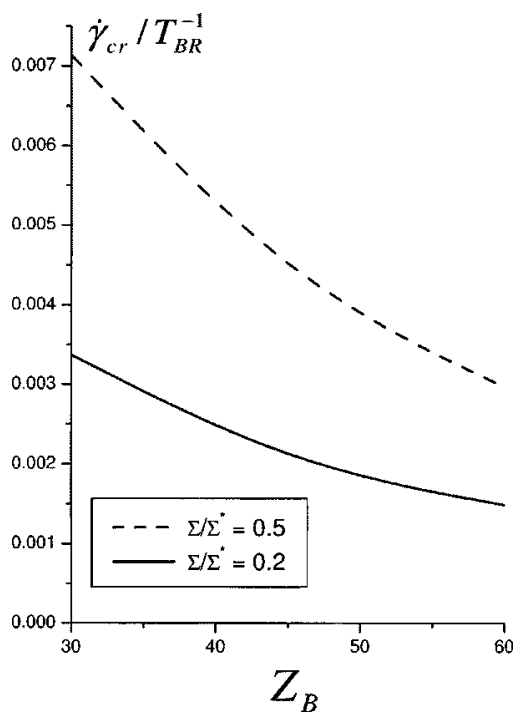

FIG. 10. Critical shear rate vs $Z_{B}$ for different grafting densities, for $Z_{T}$ $=15$.

As seen in Fig. 7, the layer thickness $h$ decreases monotonously with the shear rate $\dot{\gamma}$ which indicates that tethered chains are more oriented in faster flows. At small shear rates, the flow disturbs the coil structure of tethered chains only slightly so that $h$ is nearly equal to the diameter of the coil. In this regime, $h$ decreases with $\dot{\gamma}$ only slowly. At higher shear rates, due to an increased imbalance between convection and constraint release, the slope of the curve becomes so steep that even a small increase in the upper plate velocity $V_{p}$ (or equivalently in $\dot{\gamma}$ ) leads to a dramatic decrease in $h$. In this case, tethered chains are significantly oriented by the flow, in agreement with the hypothesis by Brochard-Wyart and de Gennes. ${ }^{5}$ A further increase in $\dot{\gamma}$ leads to a collapse of the interfacial layer after which tethered chains are completely squeezed against the wall, forming a sort of "lubrication" layer between the flowing melt and the wall. The collapse of the layer implies disentanglement between bulk and tethered chains, and therefore macroscopic wall slip. The shear rate at which the dramatic decrease of $h$ with $\dot{\gamma}$ is observed can therefore be associated with the critical shear rate $\dot{\gamma}_{\text {cr }}$ for the onset of macroscopic.

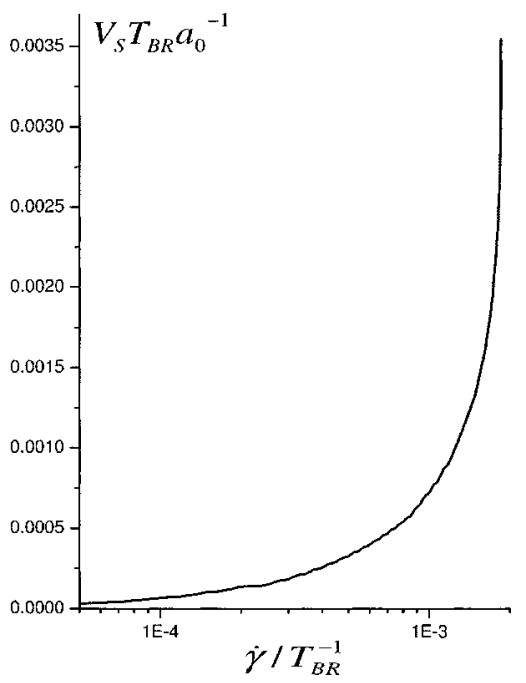

FIG. 11. Slip velocity vs shear rate, for $Z_{B}=40$ and $Z_{T}=10$.

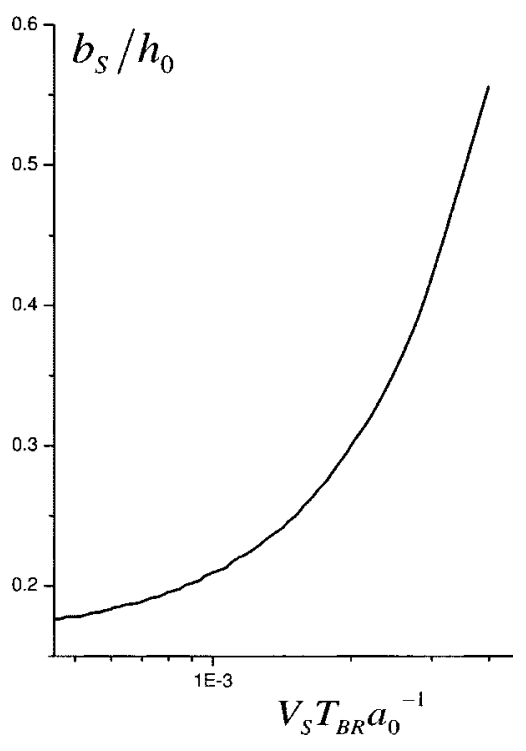

FIG. 12. Slip length vs slip velocity, for $Z_{B}=40$ and $Z_{T}=10$.

Attention must be paid that, as seen in Fig. 7, for a given shear rate $h$ is a nonmonotonous function of the surface density of tethered chains $\Sigma_{T}$. This can be explained as follows. At large $\Sigma_{T}$, where interactions between separate tethered chains suppress constraint release, tethered chains are more easily oriented by the flow than in the mushroom regime. On the other hand, for a given shear rate $\dot{\gamma}$, in the mushroom regime tethered chains undergo a larger drag force compared to higher surface densities where this drag force is distributed among a larger number of tethered chains. So for a given $\dot{\gamma}$, tethered chains are less oriented in the intermediate grafting regime (see Fig. 3) in comparison to the mushroom regime.

In Figs. 8 and 9, the critical shear rate for wall slip $\dot{\gamma}_{\mathrm{cr}}$ is presented versus $\Sigma_{T}$ for different molecular weights of bulk and tethered molecules. We remind that the mean number of constraints per chain $Z$ is defined as the ratio $M_{n} / M_{e}$, where $M_{n}$ is the average molecular weight, and $M_{e}$ is the average

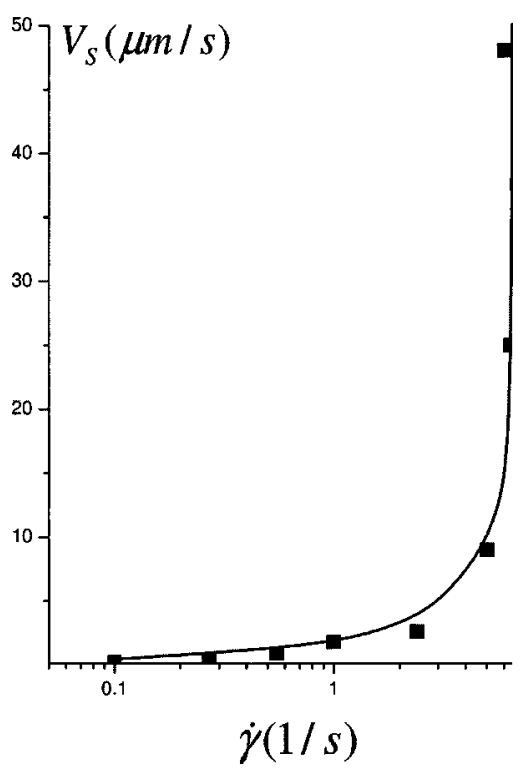

FIG. 13. Slip velocity vs shear rate $\dot{\gamma}$. The solid line is the model prediction. 


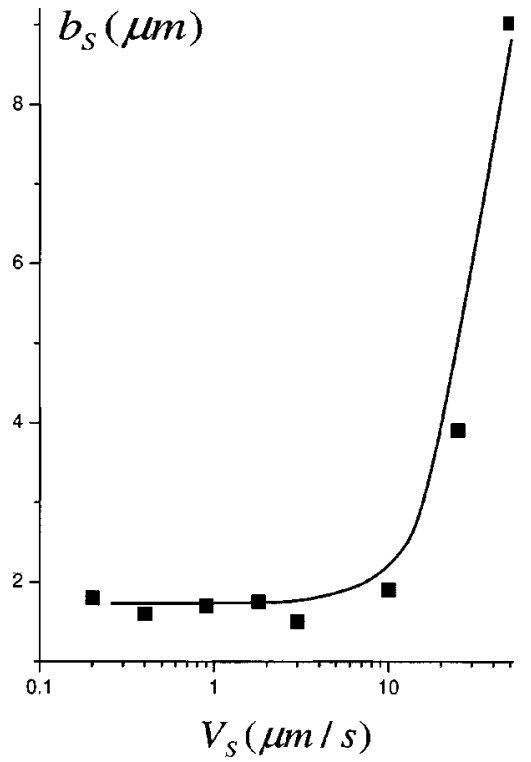

FIG. 14. Slip length vs slip velocity. The solid line is the model prediction.

molecular weight between entanglements. It is seen that, similar to the volume fraction of bulk-tethered entanglements $\psi_{B T}^{0}$ (see Figure 6), $\dot{\gamma}_{\mathrm{cr}}$ is a nonmonotonous function of $\Sigma_{T}$. In the mushroom regime, both $\psi_{B T}$ and $\dot{\gamma}_{c r}$ are small, which indicates that a small number of bulk-tethered entanglements is insufficient to prevent the onset of slip at high shear rates. Here both $\dot{\gamma}_{\mathrm{cr}}$ and $\psi_{B T}^{0}$ are proportional to $\Sigma_{T}$ which implies that separate tethered chains move independently of each other. Clearly, in this case each tethered chain gives a separate contribution to $\dot{\gamma}_{\mathrm{cr}}$. The inclusion of one new tethered chain in the interfacial layer will create new $Z_{T}$ bulk-tethered entanglements and therefore will "improve" the resistibility of the layer to the flow. A decrease in $\dot{\gamma}_{\text {cr }}$ with $\Sigma_{T}$ at high surface densities of tethered chains can be associated with that of the volume fraction of bulk-tethered entanglements in the layer $\psi_{B T}^{0}$ (see Fig. 6) and mean fraction

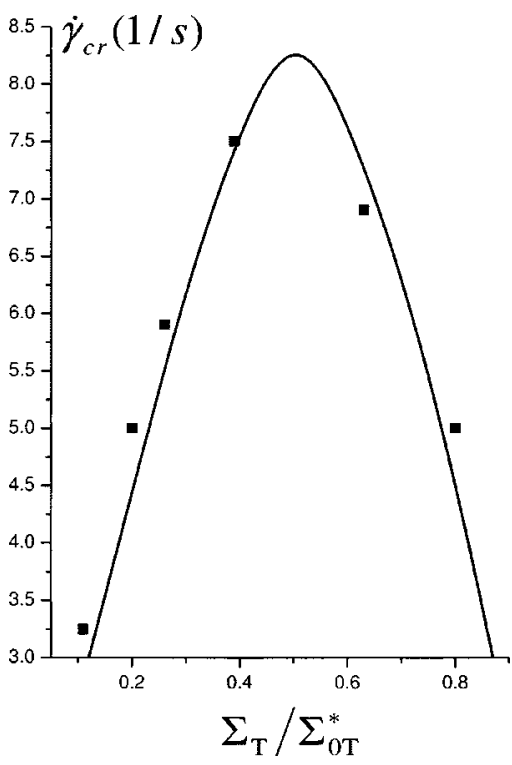

FIG. 15. Critical shear rate $\dot{\gamma}_{\text {cr }}$ vs grafting density. The solid lines are the model predictions. of bulk constraints per tethered chain $\phi_{Z}$ (see Fig. 4). In other words, by increasing $\Sigma_{T}$ we decrease the number of bulk-tethered entanglements in the layer as well as the "strength" of constraint release on tethered chains, thus facilitating an "early" stick-slip transition.

The comparison of Fig. 6 with Fig. 8 yields that the critical surface density $\Sigma_{T}^{(\mathrm{cr})}$, at which the critical shear rate $\dot{\gamma}_{\text {cr }}$ is maximal, is equal to the surface density of tethered chains at which the number of bulk-tethered entanglements in the interfacial layer is maximal. Apparently, $\Sigma_{T}^{(\mathrm{cr})}$ pinpoints a grafting regime in which tethered-tethered entanglements start to play an important role in the dynamics of the interfacial layer. According to Fig. $8, \Sigma_{T}^{(\mathrm{cr})}$ is given by

$$
\Sigma_{T}^{(\mathrm{cr})} \approx 0.5 \Sigma_{0 T}^{*}
$$

where $\Sigma_{0 T}^{*}$ is the equilibrium surface density of the dry-brush regime [see Eq. (14)].

Figure 8 shows that an increase in the molecular weight of bulk molecules leads to a decrease in $\dot{\gamma}_{\mathrm{cr}}$ at a fixed $\Sigma_{T}$. The amplitude of the maximum seems to be nearly proportional to $Z_{B}^{-1}$. However, its position, i.e., the critical surface density $\Sigma_{T}^{(\mathrm{cr})}$, is insensitive to $Z_{B}$. In contrast, as follows from Fig. 9, $\dot{\gamma}_{\text {cr }}$ can be a decreasing, increasing, or even a nonmonotonous function of the molecular weight of tethered chains, depending on the value of $\Sigma_{T}$. The amplitude of the maximum is, however, independent of $Z_{T}$, whereas its position scales as $Z_{T}^{-1 / 2}$. The nonmonotonous dependence of $\dot{\gamma}_{\text {cr }}$ on $\Sigma_{T}$ was predicted by Joshi and Lele ${ }^{9}$ and reported in Refs. 3 and 21.

Similar to the critical shear rate for spurt $\dot{\gamma}_{\mathrm{cr}}$, the corresponding critical shear stress $\sigma_{\mathrm{cr}}$ is found to be a nonmonotonous function of $\Sigma_{T}$. At $\Sigma_{T}<\Sigma_{T}^{(\mathrm{cr})}, \sigma_{\mathrm{cr}}$ can be written as

$$
\sigma_{\mathrm{cr}}=C_{1} G_{B 0} \Sigma_{T},
$$

where $C_{1}$ is a constant independent of $\Sigma_{T}$. According to Doi and Edwards, ${ }^{14}$ the bulk elastic modulus $G_{B 0}$ is proportional to the melt temperature $T$, so Eq. (29) can be written in a form similar to that proposed by Brochard-Wyart and de Gennes. ${ }^{5}$ Basing on scaling arguments, they found that in the mushroom regime

$$
\sigma_{\mathrm{cr}} \propto \Sigma_{T} k_{B} T,
$$

where $k_{B}$ is the Boltzmann constant. In the absence of desorption, $\Sigma_{T}$ is constant so that $\sigma_{\text {cr }}$ increases linearly with $T$ over the whole range of grafting regimes. Such a linear dependence was reported by many authors (see, for example, Wang and $\mathrm{Drda}^{22}$ ). In contrast, as expected for an activation process, ${ }^{10} \sigma_{\mathrm{cr}}$ is likely to decrease with temperature in the case of slip via desorption. A temperature decrease of the critical shear stress on a low surface energy wall was reported in Ref. 23. According to Doi and Edwards, ${ }^{14}$ the Rouse time of bulk chains $T_{R B} \propto T^{-1}$. Thus, Fig. 8 shows that in the case of slip via disentanglement $\dot{\gamma}_{\text {cr }}$ also scales linearly with $T$.

In Fig. 10, the critical shear rate $\dot{\gamma}_{\mathrm{cr}}$ is shown against the molecular weight of bulk chains for different grafting regimes. Since the Rouse time $T_{B R}$ of bulk chains is proportional to $Z_{B}^{2}{ }^{14}$ Fig. 10 shows that at small $\Sigma_{T} \dot{\gamma}_{\text {cr }}$ scales as $Z_{B}^{-3.4}$, in agreement with the result of Joshi and Lele. ${ }^{9}$ Note 
that at higher $\Sigma_{T}, \dot{\gamma}_{\mathrm{cr}}$ becomes slightly more sensitive to $Z_{B}$. Durliat et $a .^{21}$ reported that $\dot{\gamma}_{\mathrm{cr}}$ scales as $M_{w}^{-3.1 \pm 0.5}$ at surface densities up to the critical value $\Sigma_{T}^{(\mathrm{cr})}$. Contrary to $\dot{\gamma}_{\mathrm{cr}}$, the present model predicts only a weak dependence of the critical shear stress $\sigma_{\text {cr }}$ on $Z_{B}$, similar to the behavior reported in Ref. 24.

In Fig. 11, the slip velocity $V_{s}$, defined as the average velocity of monomers at the top of the interfacial layer, is shown versus the global shear rate $\dot{\gamma}$. Two regimes can be discerned. At small $\dot{\gamma}, V_{s}$ is much smaller than $V_{p}$ and slowly increases with $\dot{\gamma}$. However, when $\dot{\gamma}$ approaches the critical shear rate $\dot{\gamma}_{\text {cr }}$ the slope of the curve $V_{s}(\dot{\gamma})$ becomes so steep that even a small increase in $\dot{\gamma}$ leads to a dramatic increase in $V_{s}$. A further increase in $\dot{\gamma}$ results in a sudden stick-slip transition after which $V_{s}$ "jumps" up to $V_{p}$. Above $\dot{\gamma}_{\mathrm{cr}}$, a third regime of strong slip is expected for which $V_{s}$ is of the order of $V_{p}$. In this regime the flow may become unstable, so a time-dependent solution of the constitutive equations for the bulk and for the layer is required. The existence of the three regimes in the curve $V_{s}(\dot{\gamma})$ was predicted by Brochard-Wyart and de Gennes ${ }^{5}$ and reported by Durliat et $a l^{21}$

Figure 11 shows a nonzero $V_{s}$ even at small $\dot{\gamma}$, in accordance with the data by Durliat et $a l^{21}$ So the polymer melt always slips, whatever the shear rate. The amount of slip is characterized by the amplitude of the slip velocity. Attention must be paid to the fact that $V_{s}$ remains small compared to $V_{p}$ up to the transition point $\dot{\gamma}_{\text {cr }}$ which implies that this weak slip regime can hardly be inferred from macroscopic behavior such as a slope change in experimentally measured strainstress curves. At $\dot{\gamma}_{\mathrm{cr}}$, a transition from "microscopic" slip to a regime of strong slip occurs after which $V_{s}$ is no longer small, but of the order of $V_{p}$. This can be readily detected (e.g., via a significant pressure drop in controlled shear rate experiments or even visually). As seen in Fig. 11, the transition between weak and strong slips is rather sharp, i.e., ini-

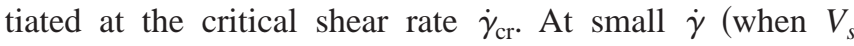
$\ll V_{p}$ ), Eq. (22) reads as

$$
\dot{\gamma}_{b}=\frac{V_{p}}{H-h} \approx \frac{V_{p}}{H}
$$

where use was made of the fact that $h$ is microscopic, whereas $H$ is macroscopic. On the other hand, the wall shear rate $\dot{\gamma}_{w}=V_{s} / h$, where $h$ is the thickness of the interfacial layer. An increase in the upper plate velocity $V_{p}$ (or, equivalently in the global shear rate $\dot{\gamma}$ ) leads to a decrease in $h$ and an increase in $V_{s}$ so that $\dot{\gamma}_{w}$ also increases. So at small shear rates both $\dot{\gamma}_{b}$ and $\dot{\gamma}_{w}$ increase with $V_{p}$. By further increasing the upper plate velocity $V_{p}$, the wall shear rate $\dot{\gamma}_{w}$ continues to grow. This dependence becomes especially strong when $\dot{\gamma}$ approaches $\dot{\gamma}_{c r}$. Then, even a small increase in $V_{p}$ leads to a sharp increase in $\dot{\gamma}_{w}$. However, as follows from Eq. (22), the sharp increase in $V_{s}$ implies that the bulk shear rate $\dot{\gamma}_{b}$ increases only slightly or even decreases with $\dot{\gamma}$ near $\dot{\gamma}_{\mathrm{cr}}$. We remind that it is $\dot{\gamma}_{w}$ that defines the rate at which tethered chains in the layer are deformed by the flow, whereas $\dot{\gamma}_{b}$ determines the resistibility of the layer to the flow via the frequency of constraint release on tethered chains $\nu_{I}$. Therefore, by increasing $V_{p}$, we increase the imbalance between convection and constraint release. At the transition point, the "weak" constraint release is no longer able to resist convection, and sustain a sufficient number of bulk-tethered entanglements in the layer to prevent the onset of macroscopic slip. This picture is consistent with the original theory of Brochard-Wyart and de Gennes ${ }^{5}$ who described a sudden loss of entanglements between tethered and bulk chains at a critical shear stress.

In Fig. 12, the slip length $b_{s}=V_{s} / \dot{\gamma}_{b}$ is shown against the slip velocity $V_{s}$. At low $V_{s}, b_{s}$ is nearly constant, in accordance with the predictions of Brochard-Wyart and de Gennes. ${ }^{5}$ The value of $b_{s}$ at small $V_{s}$ is often referred to as the zero-slip-length $b_{0} . b_{0}$ depends on a grafting regime and molecular parameters of the melt. In particular, a decrease in $\Sigma_{T}$ leads to an increase in $b_{0}$, similar to the behavior predicted by Joshi and co-workers. ${ }^{9}$ In the vicinity of the critical point a sharp increase in $b_{s}$ is observed with $V_{s}$. After a transition to strong slip, $V_{s}$ jumps to $V_{p}$ whereas $\dot{\gamma}_{b}$ decreases so that a regime of a nearly constant macroscopically large slip length is expected. The three regimes in the curve $b_{s}\left(V_{s}\right)$ were first predicted by the disentanglement model of Brochard-Wyart and de Gennes, ${ }^{5}$ and observed experimentally by Migler and co-workers (see review of Leger et al. ${ }^{3}$ ).

In the rest of this section, the model predictions will be compared with the available experimental data on slip. Leger et $a l^{3}$ and Durliat et $a l^{21}$ performed a series of experiments on monodisperse polymethylsiloxane (PDMS) chains of molecular weight of $96 \mathrm{~kg} / \mathrm{mol}$ adsorbed on a silica wall with a controlled surface density. These chains form a polymer brush which is in contact with a monodisperse PDMS melt of molecular weight of $970 \mathrm{~kg} / \mathrm{mol}$. The size and molar mass of the monomer are reported to be $0.5 \mathrm{~nm}$ and $0.074 \mathrm{~kg} / \mathrm{mol}$, respectively. Using the molecular data of Fetters et al., ${ }^{25}$ from Eq. (24) the mean entanglement spacing $a_{0}$ is estimated to be $4.8 \mathrm{~nm}$. The distance $H$ between parallel plates in the rheometer (see Fig. 5) is reported to be $8 \mu \mathrm{m}$.

In order to make a comparison quantitative, we have to specify explicitly all the parameters of the model, namely $Z_{T}$, $Z_{B}, T_{B R}$, and $\Sigma_{T}$. First, given the above molecular data, one can easily estimate the critical overlap surface density of tethered chains $\Sigma_{T}^{* *}$ as

$$
\Sigma_{T}^{* *} \approx \frac{4}{\pi N_{T} b^{2}} \approx 3.88 \times 10^{15}\left(\text { chains } / \mathrm{m}^{2}\right),
$$

where $N_{T}$ is the number of monomers per tethered molecule. On the other hand, $\Sigma_{T}^{* *}$ can be defined as the surface density at which every tethered chain has on average only one tethered constraint. From Eq. (16), where the fraction of bulk constraints per tethered chain is $\phi_{Z}=1-1 / Z_{T}$, we then have

$$
\Sigma_{T}^{* *} \approx \frac{4 h_{0}}{b^{3}} \frac{1}{Z_{T}^{2}}\left(\frac{Z_{T}}{N_{T}}\right)^{3 / 2},
$$

where $b$ and $h_{0}$ are the monomeric size and equilibrium layer thickness, respectively. The comparison of Eq. (32) with Eq. (33) yields that $Z_{T} \approx 10$, and consequently $Z_{B} \approx 100$. Although the Rouse time $T_{B R}$ of bulk molecules can be in principle measured, we will treat it as an adjustable parameter. Its value can be easily estimated from the amplitude of the maximum of the curve $\dot{\gamma}_{\mathrm{cr}}\left(\Sigma_{T}\right)$ (see Fig. 8). Comparison with 
the data of Durliat et al. ${ }^{21}$ then yields $T_{B R} \approx 1.5 \times 10^{-4} \mathrm{~s}$.

In Fig. 13, the model predictions for the slip velocity $V_{s}$ as a function of the shear rate $\dot{\gamma}$ are compared with the microscopic slip data of Leger et al. ${ }^{3}$ The surface density of tethered chains $\Sigma_{T}$ is reported to be nearly two times smaller than the corresponding critical surface density $\Sigma_{T}^{(\mathrm{cr})}$ in Eq. (28). As is seen, the model predictions are in a good agreement with the experimental data over a wide range of shear rates up to the transition point. In Fig. 14, the data of Leger et $\mathrm{al}^{3}{ }^{3}$ for the slip length versus the slip velocity are compared with the model predictions. Clearly, the present model provides a good agreement with the experimental data for both the weak slip and transition regimes. In Fig. 15, the critical shear rate for spurt $\dot{\gamma}_{\text {cr }}$ is shown versus the surface density of tethered chains $\Sigma_{T}$. The model predictions are compared with the data by Durliat et $a{ }^{21}$ As is seen, the model is able to predict $\dot{\gamma}_{\text {cr }}$ over a wide range of grafting regimes, including those where interaction between neighboring tethered chains plays an important role in the dynamics of the interfacial layer.

\section{CONCLUSIONS}

A molecular model for slip is developed which is capable to quantify the stick-slip law given the molecular and surface parameters, and the extruder geometry. Contrary to the existing scaling models for slip, it allows to write down directly a quantitative equation of motion for the local stress. The final constitutive equations have a simple structure and do not require costly numerical calculations. They allow accurate inclusion of interactions between tethered chains at high surface densities. The numerical analysis of the final system showed that both the critical shear stress and shear rate for the onset of spurt are nonmonotonous functions of the surface density of tethered chains. As explained above, the early onset of slip (and thus spurt) at low surface coverage is due to the lack of entanglements between bulk and tethered chains. On the other hand, the decrease of the critical shear rate (stress) with the surface density of tethered chains at higher densities stems from both the lack of bulktethered entanglements and suppressed constraint release. It is found that in the absence of chain desorption both the critical shear rate and stress show increase linearly with the melt temperature over a wide range of grafting regimes, in agreement with available experimental observations. The model predictions are shown to be in a good agreement with experimental data over a wide range of flow and grafting regimes.

\section{ACKNOWLEDGMENTS}

This research is supported by the Technology Foundation STW, applied science division of NWO, and the technology programme of the Ministry of Economic Affairs of the Netherlands.

${ }^{1}$ M. Denn, Annu. Rev. Fluid Mech. 33, 265 (2001)

${ }^{2}$ S. Q. Wang, Adv. Polym. Sci. 138, 229 (1999).

${ }^{3}$ L. Leger, E. Raphael, and H. Hervet, Adv. Polym. Sci. 185, 185 (1999).

${ }^{4} \mathrm{~N}$. Bergem, in 7th International Congress on Rheology, Gothenburg, Sweden (Swed. Soc. Rheol., Gothenburg, 1976), p. 50-54.

${ }^{5}$ F. Brochard-Wyart and P. G. de Gennes, Langmuir 8, 3033 (1992).

${ }^{6}$ A. Ajdari, F. Brochard-Wyart, P. G. de Gennes, L. Leibler, J. L. Viovy, and M. Rubinstein, Physica A 204, 17 (1994).

${ }^{7}$ V. Mhetar and L. A. Archer, Macromolecules 31, 6639 (1998).

${ }^{8}$ Y. M. Joshi, A. K. Lele, and R. A. Mashelkar, Macromolecules 34, 3412 (2001).

${ }^{9}$ Y. M. Joshi and A. K. Lele, J. Rheol. 46, 427 (2002).

${ }^{10}$ D. A. Hill, J. Rheol. 42, 581 (1998).

${ }^{11}$ S. G. Hatzikiriakos, Int. Polym. Process. 8, 135 (1993).

${ }^{12}$ M. A. Tchesnokov, J. Molenaar, and J. J. M. Slot, J. Non-Newtonian Fluid Mech. 126, 185 (2005)

${ }^{13}$ G. Marrucci, J. Non-Newtonian Fluid Mech. 62, 279 (1996).

${ }^{14} \mathrm{M}$. Doi and S. F. Edwards, The Theory of Polymer Dynamics (Oxford, New York, 1986).

${ }^{15}$ S. T. Milner and T. C. B. McLeish, Macromolecules 30, 2159 (1997).

${ }^{16}$ D. Mead, R. Larson, and M. Doi, Macromolecules 31, 7895 (1998).

${ }^{17}$ F. Brochard-Wyart, A. Ajdari, L. Leibler, M. Rubinstein, and J. L. Viovy, Macromolecules 27, 803 (1994).

${ }^{18}$ M. A. Tchesnokov, J. Molenaar, J. J. M. Slot, and R. Stepanyan, J. NonNewtonian Fluid Mech. 123, 185 (2004).

${ }^{19}$ A. E. Likhtman and R. S. Graham, J. Non-Newtonian Fluid Mech. 114, 1 (2003).

${ }^{20}$ P. G. de Gennes, Scaling Concepts in Polymer Physics (Cornell University Press, Ithaca, 1979).

${ }^{21}$ E. Durliat, H. Hervert, and L. Leger, Europhys. Lett. 38, 383 (1997).

${ }^{22}$ S. Q. Wang and P. A. Drda, Macromolecules 29, 2627 (1996).

${ }^{23}$ Y. M. Joshi, P. S. Tapadia, A. K. Lele, and R. A. Mashelkar, J. NonNewtonian Fluid Mech. 94, 151 (2000).

${ }^{24}$ S. Q. Wang and P. Drda, Macromolecules 29, 4115 (1996).

${ }^{25}$ L. Fetters, D. J. Lohse, D. Richter, T. Witten, and A. Zirkel, Macromolecules 27, 4639 (1994). 